\title{
EXPRESSING LESSER RELEVANCE IN ACADEMIC CONFERENCE PRESENTATIONS
}

\author{
Diana Yankova ${ }^{1}$ and Irena Vassileva ${ }^{2}$ \\ 1,2New Bulgarian University, Sofia, Bulgaria
}

Abstract

While marking importance and relevance in academic discourse has been a widely researched topic, markers of lesser significance have so far been understudied. The article therefore focuses on some of the discoursal means of expressing lesser importance in conference presentations. The corpus of the study comprises recordings of 20 presentations in English at international linguistics conferences by speakers of various cultural and linguistic backgrounds. The approach follows Deroey \& Taverniers's (2012) study of lecture discourse, whereby depending on the way lesser importance is expressed the markers are grouped under five categories. Their methodology is checked against the data provided by the transcriptions of the conference recordings to ascertain the extent to which it is applicable to other spoken academic genres. The ultimate objective is to provide steppingstones for interpreting information and distinguishing between what is important and relevant and less so in conference presentations, as well as for identifying presenters' motivation for employing this type of metadiscourse.

Keywords: metadiscourse, lesser relevance markers, conference presentations

\section{Article history:}

Received: 20 June 2020

Reviewed: 11 August 2020

Accepted: 30 November 2021

Published: 30 December 2021

\section{Contributor roles \\ Conceptualization, Investigation, Validation D.Y. (lead) Supervision, Project Administration, Data Curation, Formal Analysis (I.V.) lead; Methodology, Visualization, Writing - original draft, Writing - review and editing D.Y., I.V. (equal)}

Copyright (C) 2021 Diana Yankova and Irena Vassileva

This open access article is published and distributed under a CC BY-NC 4.0 International License which permits non-commercial use, distribution, and reproduction in any medium, provided the original author and source are credited. Permissions beyond the scope of this license may be available at dyankova@nbu.bg. If you want to use the work commercially, you must first get the authors' permission.

Citation: Yankova, D. \& Vassileva, I. (2021). Expressing Lesser Relevance in Academic Conference Presentations. English Studies at NBU, 7(2), 127-146. https://doi.org/10.33919/esnbu.21.2.1

Diana Yankova, PhD, is Professor of Linguistics and current head of the Languages and Cultures Department, New Bulgarian University, Sofia. She teaches graduate and undergraduate courses in text linguistics, translation of legislative texts, American and Canadian culture studies. She is the author of several monographs and numerous articles on legal language with special emphasis on culture and genrespecific characteristics of Common law and Continental legislation, points of convergence between legal studies and linguistics, terminological and structural considerations in translating supranational law, approximation of legislation, teaching EALP.

E-mail: dyankova@nbu.bg

https://orcid.org/0000-0003-4524-882X

Irena Vassileva, PhD, is Professor of English and German and has worked extensively on spoken and written academic communication in English, German and Bulgarian. She has published three monographs and a number of articles in peer-reviewed journals and collections of articles. Vassileva has also received various research awards from outstanding foundations in international competition and has worked at universities in Bulgaria, Germany, and the UK.

E-mail: ivasileva@nbu.bg

https://orcid.org/0000-0002-0150-6375 
The metadiscursive feature of relevance marking allows conference presenters to organize academic discourse in terms of importance and to express the evaluative stance of the speaker regarding the propositional and interpersonal content of their communication. In this way, they guide the listener into unpacking correctly the information in their talk, providing signposts as to what is more and what is less significant. Relevance marking is thus an important aspect of spoken discourse, which often has to be construed instantly on the spot.

Discourse signals are intended to help the listener predict the nature of upcoming ideas and information, and a participant who is unable to recognize these signals will be faced with additional cognitive processing demands, having to deduce both the intrinsic meaning of propositions and make inferences about the relations between them. Linguistic devices for expressing lesser relevance in discourse can also "be used to indicate a desire to close a topic, to yield a turn, or to mitigate potential face threats" (Deroey \& Taverniers, 2012, p. 2085). Therefore, these devices should be treated both as modal and interpersonal.

In the general case, a conference presentation audience consists of specialists in (often) quite a narrow field, but they may also include MA students, as well as doctoral students. At the same time, the language of an ever-increasing number of academic conference presentations is English and more and more non-native speakers are presenting their research in a language which is not their native tongue. The latter creates even more difficulties for both presenters and audiences, including such that involve the use of relevance markers.

\section{Background}

However important their function is, markers of lesser relevance in discourse have not been extensively researched. Usually, their study forms part of investigations that focus not specifically on markers of relevance but encompass overall discourse in more general terms. For instance, Caffi (1999) explored downgrading as a mitigating device in doctor-patient and psychotherapeutic spoken interaction in Italian, where different kinds of mitigators and mitigation strategies were used. According to her, "mitigation works in a multi-layered and multi-dimensional way, simultaneously affecting a plurality of 
linguistic levels and interactional dimensions" (1999, p. 883). She proposes a tripartite classification of mitigating devices based on the three components of the utterance on which downgrading can operate: the proposition, the illocution, and the utterance source, namely bushes, hedges, and shields.

Mauranen (2004) studied the epistemic and strategic uses of hedging grounded on data excerpted from the Michigan Corpus of Academic Spoken English. In their study of everyday conversations following Sperber \& Wilson's (1991) Relevance Theory, Jucker et al. consider vagueness as an interaction strategy and explore the different communicative tasks in which vague expressions are employed, whereby they "may serve as focusing devices, directing the hearer's attention to the most relevant information" (2003, p. 1765).

Basing their study on previous research by Biber, Conrad \& Cortes (2004), Nesi \& Basturkmen (2006) attempt to categorize lexical bundles in 160 university lectures and highlight their discourse-signalling role. One of their conclusions is that the lexical bundle a little bit is used to help maintain face, to downplay the task imposition, or to mitigate a potential threat to the audience's negative face.

In his study of discourse markers Fraser (2009) proposes four types of pragmatic markers: basic, commentary, discourse markers and discourse management markers and examines the three uses of the latter, while Lin (2010) explores the intensifying and softening function of pragmatic force modifiers: "From the textual perspective, the intensifying and softening effects indicate a piece of information playing either a superordinate or subordinate role and hence give rise to a logical relation between the preceding and the following utterances." (Lin, 2010, p. 1177).

More recent studies on metadiscourse markers of mitigation are most frequently based on Hyland's definition of metadiscourse as "the cover term for the self-reflective expressions used to negotiate interactional meanings in a text, assisting the writer (speaker) to express a viewpoint and engage with readers as members of a particular community" (Hyland, 2005, p. 37). However, Hyland also notes that metadiscourse is an open-ended set of language items, where not all of them perform metadiscourse roles. Hyland and Tse propose three key principles of metadiscourse, namely: 
1. that metadiscourse is distinct from propositional aspects of discourse;

2. that the term 'metadiscourse' refers to those aspects of the text that embody writer-reader interactions;

3. that metadiscourse distinguishes relations which are external to the text from those that are internal. (Hyland \& Tse, 2004, p. 159)

In terms of the first point which is of relevance for the present research, Hyland \& Tse (2004, p. 161) clarify that: "we blur the unhelpful distinction between 'primary' propositional discourse and 'secondary' metadiscourse and seek to recover the link between the ways writers intrude into their texts to organize and comment on it so that it is appropriate for a particular rhetorical context".

In view of the above, we shall argue here that markers of lesser relevance in presentations may have some of the functions of hedges, but, apart from that demonstrate also references to the nature of the proposition with regard to the specific audience and the genre.

Markers of lesser importance in conference presentations has so far been a topic that has not been often the focus of academic research. Indeed, even studies on conference presentations in general have not been abundant. Most focus on written academic genre (Hood \& Forey 2005; Campagna 2009, among many others), where there is a bulk of studies contrasting academic writing in English and another language (cf. Huemer, Lejot \& Deroey, 2019, for an overview of literature). Hyland's (2017) article, for instance, presents a large number of publications focused on the notion of "metadiscourse". Relatively more existent studies focus on the genre of academic lectures (Formentelli, 2017; Cassens \& Wegener, 2018; Zare \& Keivanloo-Shahrestanaki, 2017, to name just some more recent ones).

Unfortunately, the text and context of conference presentations has been the focus of just a handful of studies (cf. Ventola, Shalom, \& Thompson, 2002, Hood \& Forey 2005; Rowley-Jolivet \& Carter-Thomas, 2005; Mariotti 2012). The fact that spoken academic genre has been an under-researched field may be attributed to its (falsely) perceived less importance as well as to the difficulties in accessing and processing oral discourse samples. The difficulties in question are related to the necessity to ask presenters for 
permissions to record their contributions and the amount of time required for transcribing and processing, especially considering all kinds of background noises that create further complications. However, with the recent ongoing rise of the number of conferences conducted online, whose recordings can be freely accessed, some of these issues have been resolved. The present study also makes use of data excerpted from such sources.

\section{The genre of conference presentation}

It is undeniable that the conference presentation is one of the main spoken genres that a researcher is required to master at the beginning of their career since academic conferences provide the primary fora for expounding theories and showcasing research results. Perfecting the text and context of conference presentations will facilitate the swift and effective entry of a novice researcher, whether a native or a non-native speaker of English, into the respective discourse community. According to Mauranen (2001), the initiation of novice academics is effectuated more through the spoken varieties of the academic genre, since a great bulk of academic interaction predominantly occurs in an oral form, e.g., seminars, lectures, among others.

Conference presentation discourse poses a cognitive challenge since it is dense, compressed and contingent on real time construal and decoding of meaning, and thus more difficult to unpack than written academic discourse. In this hard decoding procedure, the capability to discern the relevant and less relevant chunks of information is exceedingly important. In addition, the language of international academic conferences is predominantly English, so what is needed is not only familiarity with the context of conference presentations, but also the linguistic form it adopts.

If we apply the widely embraced definition of genre by Swales (1990) as a communicative event characterized by a specific communicative function which occurs in a socio-cultural context, then the genre of the academic conference presentation can be said to be a type of research genre, originating within an academic discourse community. It carries information meant to be imparted to a specialist audience with considerable shared expertise. The conference presentation is performed live within a limited time whereby presenters have to convey not only facts and figures but also 
perform the rhetorical function of persuading the audience to accept the arguments put forth. In addition, the successful completion of the communicative act presupposes the adoption of successful interpersonal strategies and the establishment of rapport between presenter and audience.

Although the research conference presentation as a genre can be said to have "the communicative purpose of reporting research to a peer audience verbally", the features of the communicative process of delivering a presentation are hard to define precisely due to the large diversity of relative rhetorical and discursive aspects: the process is often multimodal and "appropriates other discourses and genres" (Campagna, 2009, p. 375). Mariotti (2012, p. 76) compares the genre characteristics of academic conference presentations to university lectures, finding similarities in the surface linguistic structure, in the possibility for interaction with the audience and in the potential face threats for the producers of discourse, and differences in the academic skills: in lectures it is expected for the speaker to be prepared to answer questions at any time, to elucidate or paraphrase basic notions; in conference presentations questions are usually posed by members of the audience at the end and rarely on key concepts and terminology clarification. Besides, unlike questions posed during or after lectures (which are mainly of clarifying character), the Q\&A sessions following conference presentations could be very confrontative (see Vassileva, 2005; Vassileva, 2009 for an analysis of the rhetoric of conference discussion sessions).

On the other hand, Ventola (1999) operates with the term 'semiotic spanning' to account for "how texts relate to each other by spanning semiotically, i.e., linking up with various kinds of existing and experienced texts (and other semiotic modalities) and creating new semiosis through these links" (p. 102). Analysing conference presentations and discussions, she concludes that neither the notion of cohesion, nor the notion of coherence can explain what really happens in conferencing. The genre and register model, on their part, "do not seem to capture all the aspects of this kind of linking" (Ventola, 1999, p. 111) since, for example, the presentation and the following discussion are supposed to be treated as separate genres, but they are indubitably semiotically linked. 
Differences are also observed in the communicative purpose of both genres. The university lecture is longer, usually part of a course, and is intended for students who, as a rule, do not have the same level of subject knowledge, nor the same set of skills to interpret this knowledge as the lecturer herself does; therefore, the material taught is presented in separate chunks, which need to be connected and interpreted logically and coherently associated with topics that have already been discussed within the same lecture course (or other courses). The communicative purpose of the academic conference presentation genre is to impart or share knowledge or skills mainly with an expert audience, which entails that speaker and audience share similar insight of the presentation topic. It is also a single occurrence, of shorter duration and not part of a cycle. Conference presentations are usually related to a general, pre-defined topic, and the audience has certain expectations as to the content of each individual presentation. If these expectations are flouted by a presenter, there is a danger of a communication breakdown or, at least of the presenter being ignored.

According to Mariotti (2012, p. 77), the variation in the communicative purpose of both genres gives rise to different functions of the same surface structure elements, namely discourse markers, used as guiding the audience in a topic they are knowledgeable about in conference presentations and performing the didactic function of clarifying the meaningful interrelations between notions to students who are yet to become experts in university lectures.

In the terms of Ede and Lunsford (1984) who view audience as known (audience addressed) and as a fictional creation (audience invoked), audience representation in lectures seems to be closer to 'audience addressed', as lecturers usually get to know their students or at least have clear expectations of their background knowledge and experience. In spite of real-time, face-to-face communication conference presentation audiences tend to be more 'audience invoked', since the presenter can only make assumptions about the level of expertise of the listeners. Lunsford \& Ede revisit their primary dichotomy later on $(1996,2009)$ in view of the drastic changes new technologies posed on academic communication, and expanded their model to spoken communication as well by introducing a new version of the "rhetorical triangle," the set of relationships among text, author, and audience out of which meaning grows" (Lunsford \& Ede, 2009, 
p. 8). In spite of the new challenges of the multiplicity of channels, participants and reciprocity of communication, the authors still maintain, quoting their study from 1984, that "a productive way to conceive of audience "is as an overdetermined or unusually rich concept, one which may perhaps be best specified through the analysis of precise, concrete situations" (1984, p. 168)" (2009, p. 7).

The genre of the academic conference presentation is such a concrete situation that it demonstrates a high degree of variation: firstly, in the type of presentation, which can take a different shape: a workshop, a seminar, a parallel paper or a plenary presentation. Secondly, the conference presentation can be based on research that has already been published in an academic article or an academic book or it can focus on a topic that is still at the stage of being researched. Thirdly, the audience can also range in numbers - large/small, and in expertise - experts/novice researchers/mixed.

Nevertheless, academic conference presentations have common discourse characteristics, displaying hybridity in their genre:

In most instances, for example, the oral performance is strongly associated with the development of a parallel written text. As such, a spoken conference presentation is likely to be a highly reflective text with many features that correspond to written research writing. On the other hand, there is an immediacy of audience in time and in place. This suggests a pressure in the other direction, towards a more interactive text, as the writer shapes the message in ways intended to connect with the immediate context. (Hood \& Forey, 2005, p. 292)

Whatever the diversity that can be found in academic conference presentations, they can be said to "reproduce, although with some variations, the Introduction, Materials and Methods, Results, Discussion, Conclusion (IMRDC) pattern observed in research articles" (Mariotti, 2012, p. 67). After considering some of the most outstanding features of conferencing as compared to lecturing, the two probably most important and influential spoken academic genres, we shall move on to the sample study of means of expressing lesser relevance in presentations.

\section{Aims of the study}

The present study focuses on some discoursal means of expressing lesser importance. One of the aims is to elicit clues for interpreting information and distinguishing between what is important and relevant and less so in the delivery of 
conference presentations. The second aim is to discuss some theoretical issues regarding the status of these devices as discourse markers, namely whether they should be classified under the umbrella term of 'hedging', or as possessing functions beyond the traditional understanding of that term.

\section{Methodology}

\section{Corpus}

The corpus of the study comprises the transcripts of 20 video-recorded and uploaded on the Internet academic conference presentations given at international linguistics conferences by speakers of various cultural and linguistic backgrounds with a total duration of approximately 20 hours. The presentations were of different length: some were plenaries of up to 1.5 hours, while others were regular talks of about 20 mins. Due to materials availability constraints, no distinction is made in the present study between native and non-native speakers. Besides, we believe that such a distinction is hardly viable at present, since English-medium international conferences are attended by both native and non-native speakers and in addition, there are a number of non-native scholars who are based in English-speaking countries or research institutions. Also, only verbal discourse is taken into account, since visual, prosodic and non-verbal aspects of discourse will be the object of further study.

\section{Methods}

The methodology of the study of how lesser importance is expressed in academic conference presentation draws on the model of Deroey \& Taverniers' (2012) five types of marking lesser relevance in lectures with regard to the way they indicate lesser importance. All examples in this section are by Deroey \& Taverniers.

The first group is Message status. The purpose of those markers is to either draw the line between more or less important information or to designate a chunk of discourse as having a negative value. Examples of such use are the following sentences: ....that kind of detail doesn't matter; ...it is totally irrelevant for what I am going to tell you.

Next is Topic treatment. These devices mark aspects or topics that are not covered, or such that have merited little time. Examples are: I'm going to skip the ... equation, I don't propose to go into any great depth. 
The third group of markers are those that signal Lecturer knowledge, comprising instances when the speakers impart that their knowledge is insufficient or incomplete, as in: I don't know if any of you have seen The Child Thief. I forget what is the average survival...

Assessment is the fourth function that is proposed, pertaining to lectures. These are markers signalling that something will not be assessed during exams or does not need to be studied, as in the following examples: ....it won't come up on an exam paper. ...please forget the titles and the dates 'cause they are absolute no use to you...

And finally, the fifth group is Attention and note-taking directives. Resorting to such markers, the speaker overtly and unequivocally instructs the audience to disregard what follows. Examples include: ...don't worry about the details..., I'll be giving you a handout... so you don't need to write these down.

As Deroey \& Taverniers (2012, p. 2088) rightly point out, the categories delineated above are not so definitive and may overlap. This ensues from the fact that, apart from clear instances of markers that invariably express lesser relevance (such as not important, not significant, etc.), in most cases it is achieved either covertly or summatively. The interpretation of markers as having the pragmatic function of expressing lesser relevance is also dependent on their place, their behaviour and the presence of other markers in the immediate environment, as well as on the characteristics of the genre, including its communicative purpose against which the continuum of relevance/less relevance can be assessed.

\section{Hypotheses}

Owing to the communicative purpose of conference presentations, namely, to introduce new research and findings to (mostly) expert audience, sometimes even more knowledgeable than the presenters themselves, it is only to be expected that markers of lesser importance (henceforth MLIs) would be used to downplay statements that would be considered too imposing or imperative (assertive) in their affirmation by the specialist audience, or, alternatively, would be with a low degree of significance for the expression of the intended content. 
In terms of the first option, it might be expected that the presenter's viewpoint would be displayed through the medium of hedging devices whose function is to highlight lesser relevance or undermine the significance of the results or achievements of the study reported. This is in keeping with the study by Heino et al. (2002), whereby in their corpus of conference presentations they found that more resort is made to markers of lesser importance (hedges and approximators) than to markers of importance (emphatics). However, this corpus consisted of only three presentations of 20 mins in length each. Moreover, in the classification of discourse markers the authors did not devote a special place to markers of lesser relevance outside the scope of hedging devices per se.

The present study focuses on the second type of markers that indicate the presentation of superfluous information, deviations that mark the discourse as irrelevant, or demonstrate speakers' insecurity concerning their background knowledge.

\section{Results and discussion}

\section{General observations}

We shall start the discussion of the results with an overview of the employment of MLIs in the corpus and their distribution in terms of the five categories described above.

The table below shows the absolute number of occurrences of the five categories of MLIs in the corpus:

Table 1

Markers of lesser importance - statistical results

\begin{tabular}{lc}
\hline Categories & Number of occurrences \\
\hline Message status & 0 \\
\hline Topic treatment & 12 \\
\hline Lecturer knowledge & 8 \\
\hline Assessment & 0 \\
\hline Attention and note-taking directives & 0 \\
\hline
\end{tabular}

The simple statistics presented in the table demonstrates clearly the very low degree of occurrence of MLIs, namely only 20 in 20 hours of recordings, which would roughly mean 1 per hour. Besides, in $50 \%$ of the talks there are no occurrences, while 
three of the five categories (Message status, Attention and note-taking directives, and Assessment) are not observed in the presentations at all. As the table shows, there is prevalence of MLIs in the category of Topic treatment while MLIs referring to Lecturer knowledge are present only to a certain degree.

There is a strong tendency in the corpus towards the use of markers of importance - an observation that was made in the process of transcription but will not be discussed here in more detail. We assume that the low degree of occurrence of MLIs is due to several reasons stemming primarily from the nature of the genre. The lack of the category 'Message status' could be attributed to the speakers' desire to focus on the most important points and avoid the inclusion of superfluous information, thus averting cognitive processing load. Besides, time constraints lead to the necessity to avoid deviations and concentrate instead on personal or team achievements and novelty. Presenting new information is also expected to meet the discourse community expectations when English is used as a lingua franca, namely, to adhere to linearity in the development of the argumentation (for details on linearity in academic writing in English see Clyne, 1987).

The absence of the MLIs categories 'Attention and note-taking directives' and 'Assessment' is directly related to the nature of the audience, as these devices are intended to navigate students (non-experts) in the flow of speech of a lecture and help them distinguish between relevant and less relevant information in terms of content, as well as in terms of future significance for their assessment. The audiences of conference presentations are expected to be both experts in the field and expert listeners who are fully capable of identifying the important points in a talk without this kind of explicit guidance. Moreover, depending on the specific interests of the individual participants, there may be variations in their focus of attention that should not be dictated by the speaker, since this may be felt as imposing and even arrogant. Needless to say, the category of 'Assessment' is totally irrelevant in a conference environment.

\section{MLIs in 'Topic treatment'}

Several reasons were identified for the use of Topic treatment MLIs in the corpus. Firstly, to point at limitations of topic treatment in the presentation: 
Ex. 1. So, first of all I'd like to precise that this is about a media buzz, so it is not a linguistic study of some very old and important phenomena, it's just a media buzz that occurred in 2017.

In Example 1 the speaker specifies the scope of the presentation by saying what it is not' in order to pre-empt possible expectations on the part of the audience, at the same time downplaying the nature of the research ('it's just'). This is an insurance strategy against possible criticism, but it may also be applied in cases where the previously announced title of the talk may seem too demanding or general and imprecise. The presence of inaccurate titles in conference programmes is usually due to another specific feature of conferencing, namely that such events are announced well in advance and prospective participants often tend to submit titles and abstracts based on unfinished or planned work, while the resulting presentation may turn out to diverge from the speakers' original intentions. This is another difference from lectures where such a strategy is generally unnecessary since lectures are usually parts of a planned sequence of talks and improvisations are unlikely to occur.

Secondly, MLIs in this category are used in order to clarify the focus of the presentation:

Ex. 2. This is a paper on French inclusive writing, it is essentially trying to cover the questions but not answering and saying we are for, or we are against, when we finish you vote.

Here the speaker states clearly what the intention of the presentation is, namely, only to raise but not to answer questions, thus capturing the attention of the listeners and involving them in the process of argumentation ('when we finish you vote'). The very repetition of the first-person plural 'we' and the direct address 'you' reflect a high degree of audience involvement desired by the presenter - a strategy frequently observed in conference language but hardly used in written academic texts (for a detailed analysis of the use of personal pronouns in conference language see Vassileva, 2006). At the same time, this strategy may be observed in lectures to attract students' attention and keep them interested. However, it may also happen that the lecturers eventually answer the questions themselves, which turns them into rhetorical questions. 
Thirdly, MLIs are employed in order to mark a deviation from the main topic:

Ex. 3. And I think we will, even though it is not the main talk, see examples of this in the next minutes.

In this example the speaker explicitly states that she is going to deviate from the linear development of the topic which is expected by the audience in a presentation in English. In some cases, the speaker would resort to including digressions to present results that may seem marginal for the particular topic but prove to be fruitful for future research. Thus, depending on the reactions from the audience, the presenter may decide to pursue the issue(s) further, or to drop them off. Along these lines, conferences could serve as litmus tests for scholars to establish whether they are on the right track with their studies and in line with current research in their field.

Such deviations are hardly expected in writing since they would not conform to the established conventions and may even be a reason for rejecting the publication of an article, for instance. In lectures, on the other hand, digressions could even be necessary for the elucidation of certain points that have apparently remained unclear to the students.

\section{MLIs in 'Lecturer knowledge'}

Such MLIs in the corpus point to admitting lack of or insufficient lecturer knowledge. Firstly, speakers may admit lack of general or theoretical knowledge:

Ex. 4. I have to begin with a confession. I know nothing about the Bible, the Christian Bible.

Here the speaker declares in a straightforward way their lack of familiarity with a book that is largely considered to be a must not only within the Christian communities of the world. Startling as it may be for the audience, this 'confession' made at the very beginning of the presentation not only attracts attention, but also insures the speaker against possible criticism.

Such openings would be unthinkable in both written academic English and in lectures - in the first case due to the existing conventions, in the second - because a lecturer standing in front of students would hardly admit to a knowledge gap. 
Secondly, presenters may concede lack of factual knowledge:

Ex. 5. On a tweet tweeted a few days ago by, well, I assume a girl, but actually I don't know, for all I know it could be anybody.

Ex. 6. He published that in Prague, probably he didn't want it to be read round here, but I don't know.

In the above examples, the speakers first make certain assumptions of whether something may be the case in the real world and outside the particular text of the presentation, and then eventually admit that they actually "do not know" whether it is true or not. Although this strategy may be treated as an insurance strategy, it seems more likely that the speakers simply make short remarks about information that is not of crucial importance for their argumentation. Again, as in Example 4, and for the same reasons, such acknowledgements of failure to be acquainted with facts is impossible to appear in either written academic texts or in lectures.

\section{Conclusions}

Contrary to our expectations, conference presenters very seldom mark their discourse as irrelevant. This seems to be primarily due to the variables in the communicative situation: purpose, audience, speaker roles, etc., leading to different distribution of discourse functions. When compared to lectures, conference presentations differ practically along all these parameters. In terms of purpose, while lectures aim at imparting knowledge to students who are novices in the field, presentations introduce newly acquired knowledge. While the lecturer and the students possess different levels of expertise and are therefore of unequal status, speakers at conferences share the same or similar level of subject field competence with their audiences. Besides, while the audience of the lecture is known or predictable, the conference audience is much less so in terms of specific specialization, as well as sociolinguistic factors such as age, gender, experience and, probably most importantly, cultural and professional background. Another factor is time constraints and the near impossibility to change the content or make corrections on the spot depending on reactions from the listeners, while a lecture would allow for certain modifications that can be compensated for later in the course. 
Turning now to the classification used as a starting point in this study, the category of 'Assessment' is not referred to due to the characteristics of the genre and the nature of the audience. 'Message status' is also missing in order to avoid unnecessary information and keep the content presentation succinct. This is also directly related to the time constraints mentioned above, as well as to the necessity to stick to conventions and thus meet the expectations of the audience. 'Attention and note-taking directives' are not given also due to the nature of the audience and the specificities of the genre.

'Topic treatment' MLIs are primarily used to point out study limitations and study focus - strategies related to ensuring against possible criticism. As the examples demonstrate, although such strategies are typical of academic writing in the form of hedging, the discoursal devices used in presentations are very different and would be unthinkable in writing. However, they may be expected in lectures. Practically the same is valid for admitting 'Lack of lecturer's knowledge', which is another insurance strategy.

Thus, as the results of the present sample study show, it appears that conference presentation discourse is quite different from that of academic lectures in terms of employment of MLIs both in terms of quantity and in terms of quality. In fact, apart from the (over)use of markers of importance, estimating relevance is left to the audience since the latter is considered to have enough expertise to be able to distinguish between essential and non-essential information. The analysis also demonstrates that almost all features of the use of MLIs in conference presentations are genre-specific and are predominantly dictated by the nature of the communicative situation, and especially by the nature of the audience. Some of these features have also been observed in lectures, but none of them - in academic writing. As has been shown, the conference presentation and the lecture entail different sets of academic skills which are expected to materialize, among others, also in the choice of discourse markers.

From a theoretical viewpoint, the results demonstrate that the already traditional category of hedging which has been in the focus of most of the research on MLIs is not sufficient to account for all aspects and functions of these discourse markers in spoken academic discourse. While hedging is unanimously accepted to express interpersonal relations, MLIs also demonstrate references to the nature of the proposition in view of the particular audience and genre. Therefore, in conformity with the ideas expressed by 
Hyland \& Tse (2004, p. 175), we believe that MLIs should be treated as a fuzzy, fluid category combining the features of hedging devices (interpersonal) and expressions of modality (ideational) functions in terms of Halliday \& Matthiessen (2004).

The small-scale study presented could provide a basis for future research along several lines. A qualitative and quantitative comparison of markers of relevance versus markers of lesser relevance in conference presentations merits investigation. It would be beneficial to research similarities and differences between different languages and various subject areas in order to elicit differences among subject-specific and languagespecific academic discourse communities and the consequences of these characteristics for cross-cultural academic communication. Another line of research could focus on investigating the possible differences in the use of MLIs by native and non-native speakers of English. And last but not least, future research can be directed towards a study of whether there are more specific features in the employment of MLIs in online conferencing considering their constantly increasing number and the implications of the medium for both presenters' and audiences' behaviour and their interaction.

\section{References}

Biber, D., Conrad, S., \& Cortes, V. (2004). If you look at ... Lexical Bundles in University Teaching and Textbooks. Applied Linguistics, 25(3), 371-405. https://doi.org/10.1093/applin/25.3.371

Caffi, C. (1999). On mitigation. Journal of Pragmatics, 31(7), 881-909. https://doi.org/10.1016/S0378-2166(98)00098-8

Campagna, S. (2009). Projecting Visual Reasoning in Research Conference Presentations. In Gotti, Maurizio (Ed.), Commonality and Individuality in Academic Discourse. Peter Lang, (pp. 371-391). http://hdl.handle.net/2318/136567

Cassens, J., \& Wegener, R. (2018). Supporting Students Through Notifications About Importance in Academic Lectures. In Kameas A., Stathis K. (Eds.), Ambient Intelligence. AmI 2018. Lecture Notes in Computer Science, vol 11249. Springer, Cham. https://doi.org/10.1007/978-3-030-03062-9 18

Clyne, M. (1987). Cultural differences in the organization of academic texts: English and German. Journal of Pragmatics, (11)2, 211-241. https://doi.org/10.1016/03782166(87)90196-2 
Deroey, K., \& Taverniers, M. (2012). 'Ignore that 'cause it's totally irrelevant': Marking lesser relevance in lectures. Journal of Pragmatics, 44(14), 2085-2099. https://doi.org/10.1016/j.pragma.2012.10.001

Ede, L., \& Lunsford, A. (1984). Audience Addressed / Audience Invoked: The Role of Audience in Composition Theory and Pedagogy. College Composition and Communication, 35(2), 155-171. https://doi.org/10.2307/358093

Formentelli, M. (2017). Taking Stance in English as a Lingua Franca: Managing Interpersonal Relations in Academic Lectures. Cambridge Scholars Publishing.

Fraser, B. (2009). Topic orientation markers. Journal of Pragmatics 41(5), 892-898. https://doi.org/10.1016/j.pragma.2008.08.006

Halliday, M. A. K., \& Matthiessen, C. M. I. (2004). An Introduction to Functional Grammar. London: Routledge.

Heino, A., Tervonnen, E., \& Tommola, J. (2002). Metadisourse in Academic Conference Presentations. In Ventola, E., Shalom, C., Thompson, S. (Eds.), The Language of Conferencing. Peter Lang, (pp. 127-146).

Hood, S., \& Forey, G. (2005). Introducing a conference paper: Getting interpersonal with your audience. Journal of English for Academic Purposes, (4)4, 291-306. https://doi.org/10.1016/j.jeap.2005.07.003

Huemer, B., Lejot, E. \& Deroey, K. (2019). Academic writing across languages: multilingual and contrastive approaches in higher education. Böhlau. https://doi.org/10.7767/9783205208815

Hyland, K. (2005). Metadiscourse: exploring interaction in writing. Continuum.

Hyland, K. (2017). Metadiscourse: What is it and where is it going?. Journal of Pragmatics, 113, 16-29. https://doi.org/10.1016/j.pragma.2017.03.007

Hyland, K., \& Tse, P. (2004). Metadiscourse in academic writing: A reappraisal. Applied Linguistics, 25(2), 156-177. https://doi.org/10.1093/applin/25.2.156

Jucker, A. H., Smith, S.W., \& Lüdge, T. (2003). Interactive aspects of vagueness in conversation. Journal of Pragmatics, 35(12), 1737-1769.

https://doi.org/10.1016/S0378-2166(02)00188-1

Lin, C. (2010). '... that's actually sort of you know trying to get consultants in. ..': functions and multifunctionality of modifiers in academic lectures. Journal of Pragmatics, 42(5), 1173-1183. https://doi.org/10.1016/i.pragma.2009.10.001

Lunsford, A., \& Ede, L. (1996). Representing Audience: 'Successful' Discourse and Disciplinary Critique. College Composition and Communication, 47(2), 167-179. https://doi.org/10.2307/358791 
Lunsford, A., \& Ede, L. (2009). Among the Audience: On Audience in an Age of New Literacies. In Elizabeth Weiser, Brian Fehler, and Angela González (Eds.), Engaging Audience: Writing in an Age of New Literacies. Urbana, IL: NCTE. (pp. 42-69).

Mariotti, C. (2012). Genre Variation in Academic Spoken English: The Case of Lectures and Research Conference Presentations. In Maci, S. \& Sala, M. (Eds.), Genre Variation in Academic Communication Emerging Disciplinary Trends. (pp. 63-84). CELSB: Bergamo.

Mauranen, A. (2001). Reflexive Academic Talk: Observations from MICASE. In Swales, J. M. \& Simpson, R. (Eds.), Corpus Linguistics in North America. University of Michigan Press, (pp. 165-178). https://doi.org/10.3998/mpub.11411

Mauranen, A. (2004). “They're a little bit different”. Observations on hedges in academic talk. In K. Aijmer, \& A-B. Stenström (Eds.), Discourse Patterns in Spoken and Written Corpora, (pp. 173-198). John Benjamins. https://doi.org/10.1075/pbns.120

Nesi, H., \& Basturkmen, H. (2006). Lexical bundles and discourse signaling in academic lecturers. International Journal of Corpus Linguistics, 11(3), 283-304. https://doi.org/10.1075/ijcl.11.3.04nes

Rowley-Jolivet, E. \& Carter-Thomas, S. (2005). The rhetoric of conference presentation introductions: context, argument and interaction. International Journal of Applied Linguistics, 15(1), 45-70.https://doi.org/10.1111/j.1473-4192.2005.00080.x

Sperber, D., \& Wilson, D. (1991). Loose talk. In Davis, Steven (Ed.), Pragmatics. A Reader. Oxford University Press, (pp. 540-549). https://doi.org/10.7202/602742ar

Swales, J. (1990). Genre Analysis: English in Academic and Research Settings. Cambridge University Press.

Vassileva, I. (2005). Englisch und Deutsch als Sprachen internationaler Konferenzdiskussionen. In Eva van Leewen (Ed.), Sprachenlernen als Investition in die Zukunft. Wirkungskreise eines Sprachlernzetrums. Tübingen: Gunter Narr Verlag, (pp. 389-405).

Vassileva, I. (2006). Author-Audience Interaction. A Cross-Cultural Perspective. Asgard Verlag.

Vassileva, I. (2009). Argumentative strategies in conference discussion sessions. In Eija Suomela-Salmi, Fred Dervin (Eds.), Cross-Linguistic and Cross-Cultural Perspectives on Academic discourse. John Benjamins. (pp. 219-240).

Ventola, E. (1999). Semiotic Spanning at Conferences: Cohesion and Coherence in and across Conference Papers and their Discussions. In Bublitz, W., Lenk, U., Ventola, E. (Eds.), Coherence in Spoken and Written Discourse. How to create it and how to describe it. John Benjamins. (pp. 101-123). https://doi.org/10.1075/pbns.63.09ven 
Ventola, E., Shalom, C., \& Thompson, S. (Eds.) (2002). The language of conferencing. Peter Lang.

Zare, J., \& Keivanloo-Shahrestanaki, Z. (2017). The language of English academic lectures: The case of field of study in highlighting importance. Lingua, 193, 36-50. https://doi.org/10.1016/j.lingua.2017.04.005 